\title{
PERFIL EPIDEMIOLÓGICO DO CÂNCER DE ESÔFAGO NO BRASIL: UM ESTUDO DESCRITIVO
}

\author{
Ana Maria Lima Carneiro de Andrade', Ana Maria Correia Alencarl, Amon Vitorino Duartel, \\ Bárbara Torquato Alves ${ }^{1}$, Esther de Macedo Lira ${ }^{1}$, Kevellyn Cruz Aguilera ${ }^{1}$, Patrícia Sobral Luna Quidute ${ }^{2}$
}

Introdução: As neoplasias malignas são a $2^{\mathrm{a}}$ causa de morte por doenças crônicas não transmissíveis em todo mundo. Neste cenário as neoplasias de esôfago ganham importância estando entre as mais incidentes no sexo masculino e possuem uma alta letalidade, já que geralmente é diagnosticada em estágios tardios. Tornam-se necessários estudos epidemiológicos para proposição de meios de prevenção, rastreamento e melhores tratamentos. Objetivo: Apresentar a epidemiologia do câncer esofágico no Brasil. Método: Trata-se de um estudo de cunho descritivo, utilizando como base dados secundários disponíveis na internet. Para descrever a epidemiologia do câncer esofágico foi utilizada a estimativa da taxa de incidência e mortalidade, ajustando-se os dados de acordo com a População Mundial de 1960 para fins de comparabilidade. Os critérios de inclusão para os Registros de Câncer de Base Populacional foram: disponibilidade, qualidade e completude na temporalidade, sendo o período delimitado de 2000-2009, tais dados são compilados pelo INCA, enquanto os registros da população residente foram extraídos do DATASUS. Os dados sobre mortalidade foram reproduzidos do tabulador do Atlas de Mortalidade do INCA o período delimitado para tal foi de 2000-2015. Para cálculo de tendência foi o utilizado o software JOINPOINT. Resultados: $\mathrm{O}$ câncer de esôfago apresenta variações das taxas de incidência sugerindo que exista o envolvimento de fatores ambientais. No Brasil há uma disparidade entre os sexos e as regiões, ganhando destaque a região Sul com maior estimativa de taxa de incidência e mortalidade. Uma análise da tendência de incidência reflete uma diminuição estatisticamente não significativa para ambos os sexos em todas as regiões, excetuando-se o sexo feminino na região Sul onde há uma tendência ao aumento da incidência. $\mathrm{O}$ câncer esofágico possui uma alta letalidade, e é a $6^{\mathrm{a}}$ neoplasia com maior mortalidade no Brasil, para ambos os sexos, a análise da tendência de mortalidade permite observar variações estatisticamente significativas apenas no sexo masculino da região Sul onde houve uma diminuição na tendência da mortalidade. Conclusão: A incidência e a mortalidade do câncer de esôfago tendem a aumentar com o envelhecimento, e mesmo sendo um câncer com alta letalidade ainda não há teste de rastreamento disponível sendo preciso investir na prevenção primária de forma a evitar os possíveis fatores de risco.

Palavras Chave: Câncer de esôfago. Epidemiologia. Incidência. Mortalidade.

\footnotetext{
${ }^{1}$ Discente do curso de Medicina pela Faculdade de Medicina Estácio de Juazeiro do Norte;

2 Mestranda em Ciências da Saúde pela Faculdade de Medicina do ABC-SP e Médica Radiologista do Hospital São Vicente de Paulo.

Autor correspondente: Email: anafaculdadem30@gmail.com.
}

8 Id on Line Rev. Mult. Psic. V.12, N. 40. 2018 - ISSN 1981-1179 EDIÇÃO ESPECIAL: I CURSO DE ONCOLOGIA DO CARIRI / II JORNADA DE PESQUISA QUANTI-QUALITATIVA EM ONCOLOGIA. JUAZEIRO DO NORTE, O5 A 10 DE MARÇO DE 2018.

Edição eletrônica em http://idonline.emnuvens.com.br/id 